\title{
A Solvable Model of Two-Dimensional Dilaton-Gravity Coupled to a Massless Scalar Field
}

\author{
Marco Cavaglià* \\ Max-Planck-Institut für Gravitationsphysik \\ Albert-Einstein-Institut \\ Schlaatzweg 1, D-14473 Potsdam, Germany
}

(August 22, 2018)

\begin{abstract}
We present a solvable model of two-dimensional dilaton-gravity coupled to a massless scalar field. We locally integrate the field equations and briefly discuss the properties of the solutions. For a particular choice of the coupling between the dilaton and the scalar field the model can be interpreted as the two-dimensional effective theory of $2+1$ cylindrical gravity minimally coupled to a massless scalar field.
\end{abstract}

PACS number(s): 04.60.Kz, 04.20.Jb

\section{INTRODUCTION}

The investigation of lower-dimensional gravity is receiving a lot of attention because of its connection with string theory, dimensionally reduced models (minisuperspaces and midisuperspaces) and black hole physics [1]. Lower dimensional models may further provide some insight into the difficult challenge of quantizing gravity theories in the (more physical) four dimensional case. Hence, many $0+1,1+1$ and $2+1$ integrable models have been analyzed and solved in the literature, both from the classical and quantum points of view [2].

In this context many papers have been devoted to the discussion of integrable and non-integrable two-dimensional models [2]. It is well known that two-dimensional dilaton-gravity with an arbitrary potential is (classically) completely integrable [3]. A remarkable feature of this model is that any solution possesses a Killing vector [4], i.e. the metric tensor and the dilaton $\phi$ can be cast in the form [3]

$$
\begin{aligned}
& d s^{2}=4 \rho(u, v) d u d v, \\
& \rho(u, v)=h(\psi) \partial_{u} \psi \partial_{v} \psi, \quad \phi \equiv \phi(\psi),
\end{aligned}
$$

where $\psi$ is a harmonic function, i.e. $\partial_{u} \partial_{v} \psi=0$. (With a somewhat improper terminology we will call these solutions "static", even though the Killing vector is not timelike and hypersurface orthogonal.) This is the content of the generalized Birkhoff theorem [3, 边. When a scalar field is added to the model, the Birkhoff theorem is no longer valid and non-static solutions appear. It is then worthwile to investigate how the presence of a scalar field is related to issues like integrability and absence of Killing vectors. This perhaps can help shed light on some unsolved problems in classical and quantum gravity as, for instance, the origin of the black hole entropy.

Having this in mind, the purpose of this brief report is the discussion of the general solution of the model described by the action

$$
S=\int d^{2} x \sqrt{-g}\left[\phi R+\gamma V(\phi)(\nabla \chi)^{2}\right],
$$

where $R$ is the Ricci scalar, $\phi$ is the dilaton field, and $\chi$ is the massless scalar field. $\gamma$ is a coupling constant and we have set $16 \pi G=1$. Despite of the non trivial coupling between the dilaton and the scalar field the model is completely solvable for a large class of functions $V(\phi)$. Even though in this simple model one cannot obtain any black hole solution, the discussion of the general solution of the model based upon Eq. (1.3) is important at least for two reasons: first this is (to our knowledge) the only known example of a solvable dilaton-gravity-matter model with a non-trivial coupling between the dilaton and the scalar field; second, when $V(\phi)=\phi$ the model can be interpreted as the twodimensional effective theory of $2+1 \mathbb{R} \times S^{1}$ cylindrical gravity minimally coupled to a massless scalar field [5]. In this case the dilaton plays the role of the scale factor of $S^{1}$.

*Electronic address: cavaglia@aei-potsdam.mpg.de 


\section{FIELD EQUATIONS AND GENERAL SOLUTIONS}

From the action Eq. (1.3) it is straightforward to obtain the field equations. They can be cast in a useful and simple form writing the two-dimensional line element in the "conformal gauge" (1.1). The result is

$$
\begin{aligned}
& \partial_{u} \partial_{v} \phi=0, \\
& \partial_{u} \partial_{v}(\ln \rho)=\gamma V^{\prime}(\phi) \partial_{u} \chi \partial_{v} \chi \\
& \partial_{u}\left[V(\phi) \partial_{v} \chi\right]+\partial_{v}\left[V(\phi) \partial_{u} \chi\right]=0, \\
& \rho \partial_{u}\left(\frac{\partial_{u} \phi}{\rho}\right)=\gamma V(\phi)\left(\partial_{u} \chi\right)^{2}, \\
& \rho \partial_{v}\left(\frac{\partial_{v} \phi}{\rho}\right)=\gamma V(\phi)\left(\partial_{v} \chi\right)^{2}
\end{aligned}
$$

where the prime represents the derivative w.r.t. $\phi$. It is surprising that the field equations can be locally integrated. The equation for the field $\phi$ is the key for solving the system. From Eq. (2.1), it follows that $\phi$ is indeed a harmonic function. Therefore, the general solution of Eq. (2.1) is $\phi(u, v)=a(u)+b(v)$, where $a(u)$ and $b(v)$ are arbitrary functions. The general solution can be classified in three distinct classes: i) $\phi(u, v)=\phi_{0}$, i.e. $\phi$ constant; ii $) \partial_{u} \phi(u, v)=0$ or $\partial_{v} \phi(u, v)=0$, i.e. $\phi$ depending on a single variable; iii) $\phi$ depending on both $u$ and $v$ variables. The first two cases identify degenerate solutions of the model. Let us now discuss separately the above cases.

\section{A. Constant $\phi$}

This is the simplest (degenerate) case. When $\partial_{u} \phi(u, v)=0$ and $\partial_{v} \phi(u, v)=0$ Eqs. 2.2 2.5 reduce to (we assume $\left.V\left(\phi_{0}\right) \neq 0\right)$

$$
\partial_{u} \partial_{v}(\ln \rho)=0, \quad \partial_{u} \chi=0, \quad \partial_{v} \chi=0 .
$$

The general solution of Eqs. (2.6) is obvious

$$
\chi=\chi_{0}, \quad \ln (\rho)=c(u)+d(v),
$$

where $\chi_{0}$ is a constant and $c(u)$ and $d(v)$ are two arbitrary functions. Recalling Eq. (1.1) and using the reparametrization invariance of the metric in the $u$ and $v$ variables, the three-dimensional line element can be cast in the form $d s^{2}=4 d \zeta d v$, where $\zeta=\int d u e^{c(u)}$ and $v=\int d v e^{d(v)}$. The solution is then static and the spacetime is flat, as expected since this case corresponds to a pure dilaton-gravity model with vanishing potential. Since $\phi$ is constant, the above solution represents a flat $\left(\mathbb{R}^{2} \times S^{1}\right)$ spacetime with constant $S^{1}$ scale factor when interpreted as a $2+1$ reduced model $(V(\phi)=\phi)$.

\section{B. $\phi$ Depending on a Single Coordinate}

Let us suppose, without loss of generality, that $\partial_{v} \phi=0$, i.e. $b(v)=0$. (Alternatively, $\partial_{u} \phi=0$.) From Eq. (2.5) we have $\partial_{v} \chi=0$, and Eqs. (2.2 2.4) reduce to the form

$$
\begin{aligned}
& \partial_{u} \partial_{v}(\ln \rho)=0 \\
& \frac{d^{2} \phi}{d u^{2}}-\partial_{u} \ln (\rho) \frac{d \phi}{d u}=\gamma V(\phi)\left(\frac{d \chi}{d u}\right)^{2} .
\end{aligned}
$$

(Equation (2.3) is identically satisfied.) The general solution of Eqs. (2.8,2.9) is

$$
\rho=\frac{d \phi}{d u} \exp \left[d(v)-\gamma \int_{\phi_{0}}^{\phi} d \phi^{\prime} V\left(\phi^{\prime}\right)\left(\frac{d \chi}{d \phi^{\prime}}\right)^{2}\right],
$$

where $d(v)$ is an arbitrary function of $v, \phi_{0}$ is an integration constant, and $\chi \equiv \chi[\phi(u)]$. The two-dimensional line element then represents a flat spacetime, even though the scalar field is not constant. Note that all fields depend on a single variable $(\phi)$, however the solution $(2.10)$ is not strictly a "static" solution in the sense (1.1.1.2). (A 
similar solution arises in two-dimensional dilaton-gravity when the dilaton has vanishing potential.) The nature of the solution becomes particularly evident when $V\left(\phi^{\prime}\right)=\phi^{\prime}$ and the model is interpreted in $2+1$ dimensions. In this case the three-dimensional line element corresponding to the solution (2.10) reads $\left(V\left(\phi^{\prime}\right)=\phi^{\prime}\right)$

$$
d s^{2}=4 \exp \left[-\gamma \int_{\phi_{0}}^{\phi} d \phi^{\prime} \phi^{\prime}\left(\frac{d \chi}{d \phi^{\prime}}\right)^{2}\right] d \phi d v+\phi^{2} d \theta^{2},
$$

where we have defined the new coordinate $v$ as in the previous section and $\theta \in[0,1]$ is the $S^{1}$ variable. The geometry of the spacetime is $\mathbb{R}^{2} \times S^{1}$, and the scale factor $\phi$ is a function of the light cone variable orthogonal to $v$. For instance, choosing $\chi=\chi_{0}+\arctan (\phi / k)$, Eq. (2.11) reads $(x=v+\phi$ and $t=v-\phi)$

$$
d s^{2}=f_{0} e^{\frac{\gamma / 2}{1+(x-t)^{2} / 4 k^{2}}}\left(-d t^{2}+d x^{2}\right)+\frac{(x-t)^{2}}{4} d \theta^{2} .
$$

\section{Complete Case}

In this case, setting

$$
\rho(u, v)=f(u, v) \frac{d a(u)}{d u} \frac{d b(v)}{d v},
$$

and using the new coordinates $(a, b)$ (note that the coordinate transformation is never degenerate, the degenerate cases being included in the previous sections), equations (2.2-2.5) reduce to

$$
\begin{aligned}
& \partial_{a} \partial_{b}(\ln f)=\gamma V^{\prime}(a+b) \partial_{a} \chi \partial_{b} \chi, \\
& V^{\prime}(a+b)\left(\partial_{a} \chi+\partial_{b} \chi\right)+2 V(a+b) \partial_{a} \partial_{b} \chi=0, \\
& \partial_{a}(\ln f)=-\gamma V(a+b)\left(\partial_{a} \chi\right)^{2}, \\
& \partial_{b}(\ln f)=-\gamma V(a+b)\left(\partial_{b} \chi\right)^{2} .
\end{aligned}
$$

Now the system of second order partial differential equations (2.14 2.17) can be integrated, solving first Eq. (2.15), and then using the solution $\chi$ in Eqs. (2.16,2.17). This program can be easily completed using the new variables $z=a+b$ and $w=a-b$. Since Eq. (2.15) and the constraints (2.16.2.17) imply Eq. (2.14), we can forget the latter and write

$$
\begin{aligned}
& \partial_{z}^{2} \chi+\frac{V^{\prime}(z)}{V(z)} \partial_{z} \chi=\partial_{w}^{2} \chi, \\
& \partial_{z}(\ln f)=-\gamma V(z)\left[\left(\partial_{z} \chi\right)^{2}+\left(\partial_{w} \chi\right)^{2}\right], \\
& \partial_{w}(\ln f)=-2 \gamma V(z) \partial_{z} \chi \partial_{w} \chi,
\end{aligned}
$$

where $V^{\prime}=\frac{d V(z)}{d z}$. Equation (2.18) corresponds to Eq. (2.15) and Eqs. (2.19 2.20) are the sum and the difference of Eqs. (2.16,2.17) respectively. Finally, given a solution of Eq. (2.18), the (logarithm of the) physical conformal factor of the two-dimensional metric $f$ can be written as a functional of $\chi$ and locally cast in the form

$$
\begin{aligned}
& \ln f\left(z, w ; f_{0}\right)=\ln f_{0}+ \\
& \quad-2 \gamma V(z) \int_{w_{0}}^{w} d w^{\prime} \partial_{w^{\prime}} \chi\left(w^{\prime}, z\right) \partial_{z} \chi\left(w^{\prime}, z\right)+ \\
& \quad-\gamma \int^{z} d z^{\prime} V\left(z^{\prime}\right)\left\{\left[\partial_{w} \chi\left(w, z^{\prime}\right)\right]^{2}+\left[\partial_{z} \chi\left(w, z^{\prime}\right)\right]^{2}\right\}_{w=w_{0}},
\end{aligned}
$$

where $f_{0}$ and $w_{0}$ are two constants. Let us now focus attention on Eq. (2.18). Since the latter is a linear separable partial differential equation in the variables $z$ and $w$, the solution can be written in the form []

$$
\chi(z, w)=\chi_{0}+\int_{-\infty}^{\infty} d \lambda C(\lambda) \eta(z, \lambda) \xi(w, \lambda),
$$

where $\eta$ and $\xi$ are the solutions of the linear second order ordinary differential equations 


$$
\frac{d^{2} \eta}{d z^{2}}+\frac{V^{\prime}(z)}{V(z)} \frac{d \eta}{d z}=\lambda \eta, \quad \frac{d^{2} \xi}{d w^{2}}=\lambda \xi
$$

It can be proved that the solution (2.22) is the most general solution of Eq. (2.18) provided that the completeness theorem for both Eqs. (2.23) holds. (See for instance [7].) This rather weak assumption is satisfied for a wide class of physical, well-behaved, functions $V(z)$ in Eqs. (2.23). As a concrete example of the formalism, let us now consider the case $V(z)=z$ corresponding to the 2+1-dimensional model. The (real) solution 2.22) reads (in this case it is straightforward to verify that the completeness theorem holds because the first equation in (2.23) coincides with the Bessel/modified Bessel equation depending on the sign of $\lambda$ )

$$
\chi(z, w)=\chi_{0}+\int_{0}^{\infty} d \alpha\left\{\chi_{\alpha}^{(1)}(z, w)+\chi_{\alpha}^{(2)}(z, w)\right\}
$$

where

$$
\begin{aligned}
\chi_{\alpha}^{(1)}(z, w)= & {\left[A_{1}(\alpha) \sin (\alpha w)+B_{1}(\alpha) \cos (\alpha w)\right] } \\
\cdot & {\left[C_{1}(\alpha) J_{0}(\alpha z)+D_{1}(\alpha) Y_{0}(\alpha z)\right], } \\
\chi_{\alpha}^{(2)}(z, w)= & {\left[A_{2}(\alpha) e^{\alpha w}+B_{2}(\alpha) e^{-\alpha w}\right] } \\
\cdot & {\left[C_{2}(\alpha) I_{0}(\alpha z)+D_{2}(\alpha) K_{0}(\alpha z)\right], }
\end{aligned}
$$

where $J_{0}$ and $Y_{0}$ are the zero order Bessel functions of first and second kind, $I_{0}$ and $K_{0}$ are the zero order modified Bessel functions, and the coefficients $A, . ., D$ are real functions.

Starting from Eqs. (2.24 2.26), or directly from Eqs. (2.18 2.20), some interesting particular solutions can be calculated. Let us look, for example, for a solution of Eq. (2.18) of the form $\chi(z, w)=\zeta(z)+v(w)$. Inserting the previous ansatz in Eq. (2.18), we have

$$
\chi(z, w)=\chi_{0}+k_{1} \ln z+k_{2} w+k_{3}\left(w^{2}+\frac{z^{2}}{2}\right) .
$$

The conformal scale factor of the two-dimensional line element is

$$
f=f_{0} z^{-\gamma k_{1}^{2}} g(z, w),
$$

where

$$
g(z, w)=e^{-2 \gamma w\left(k_{1}+k_{3} z^{2}\right)\left(k_{2}+k_{3} w\right)-\frac{\gamma z^{2}}{4}\left(2 k_{2}^{2}+4 k_{1} k_{3}+k_{3}^{2} z^{2}\right)} .
$$

The solution (2.27 2.29) is generally non-static in the sense (1.1 1.2) because the fields depend on both variables. (A particular case is given by the choice $k_{1}=k_{3}=0$. In this case $\chi \equiv \chi(w)$ and $f=\exp \left(-\gamma k_{2}^{2} z^{2} / 2\right)$ depends only on $z$. Hence, according to (1.1.1.2) we have a flat spacetime but a non-static solution.) However, when $k_{2}=k_{3}=0$, i.e. $g(z, w)=1$, Eqs. (2.27 2.29) reduce to the (well-known) static solution [6]. (Alternatively, the latter can be obtained directly from Eqs. (2.24 2.26) setting, for instance, $A_{i}=B_{i}=\delta(\alpha)$.) Let us see this in detail. Recalling Eq. (1.1) and Eq. (2.13) the three-dimensional line element reads $(w=\tau, z=R)$

$$
\begin{aligned}
& d s^{2}=f_{0} R^{-\gamma k_{1}^{2}}\left(-d \tau^{2}+d R^{2}\right)+R^{2} d \theta^{2}, \\
& \chi=k_{1} \ln \left(R / R_{0}\right) .
\end{aligned}
$$

The above solution can be cast in a more familiar form with a redefinition of the integration constants and a change of coordinates. Let us set $R=\beta r^{N / 2}$ and $\tau=t M$, where $\beta$ is a parameter with dimensions of length and $N, M$ are related to $k_{1}$ and $f_{0}$ by $N=2 /\left(1+4 k_{1}^{2}\right), M=\left(2 / N f_{0}\right) \beta^{1-2 / N}$. With these redefinitions the line element (2.30) becomes $(\gamma=-4$, low-energy string case)

$$
d s^{2}=-U(r) d t^{2}+\beta^{2} U^{-1}(r) d r^{2}+\beta^{2} r^{N} d \theta^{2},
$$

where $U(r)=(2 M / N) r^{1-N / 2}$. As expected, the model does not admit black hole solutions and the metric becomes $\mathbb{R}^{3}$ flat when $k_{1}=0$, i.e. when the scalar field is constant. This can be verified directly from Eq. (2.21), recalling that $\phi$ is a harmonic function, and Eq. (2.13).

Let us briefly conclude the section with another interesting set of solutions. Choosing for instance in Eq. (2.25) $A_{1}=D_{1}=0, B_{1} C_{1}=K$, and using Eq. (2.24), we have [8] 


$$
\chi(z, w)=\chi_{0}+\frac{K}{\sqrt{z^{2}-w^{2}}},
$$

where $K$ is a constant. From Eq. (2.21) it is straightforward to obtain the metric

$$
d s^{2}=f_{0} e^{\frac{\gamma K^{2} z^{2}}{2\left(z^{2}-w^{2}\right)^{2}}}\left(-d w^{2}+d z^{2}\right)+z^{2} d \theta^{2} .
$$

The choice $B_{1}=D_{1}=0, A_{1} C_{1}=K$ gives instead the complementary solution

$$
\begin{aligned}
& \chi(z, w)=\chi_{0}+\frac{K}{\sqrt{w^{2}-z^{2}}}, \\
& d s^{2}=f_{0} e^{-\frac{\gamma K^{2} z^{2}}{2\left(w^{2}-z^{2}\right)^{2}}\left(-d w^{2}+d z^{2}\right)+z^{2} d \theta^{2} .}
\end{aligned}
$$

The discussion of the global properties of Eqs. (2.33-2.36) is beyond the scope of this brief report and will be discussed elsewhere, so here we will not enter into details. Let us stress, however, that the above solutions are asymptotically flat and singular when $w \pm z=0$, i.e. when the scalar field diverges. With different choices of the coefficients in Eqs. (2.25 2.26) is it possible to construct non singular solutions.

\section{CONCLUSIONS}

In this note we have briefly discussed the two-dimensional dilaton-gravity-matter theory described by the action (1.3). When $V(\phi)=\phi$ the model can be interpreted as the two-dimensional effective theory of $2+1$ cylindrical gravity minimally coupled to a massless scalar field.

The model of Eq. (1.3) has the remarkable property of being completely solvable and we have derived and classified its solutions for a large class of functions $V(\phi)$. It is well-known that any two-dimensional pure dilaton-gravity theory satisfies the generalized Birkhoff theorem, i.e. any solution of the system can be reduced to the form (1.1 1.2). Here, due to the presence of the scalar field, the Birkhoff theorem is no longer valid. It is then interesting to examine how the presence of the scalar field modifies the equations of motion w.r.t. to the pure dilaton-gravity case. Let us consider for simplicity $\partial_{u} \phi \neq 0$ and $\partial_{v} \phi \neq 0$. In this case Eqs. (2.1) and (2.3 2.5) imply Eq. (2.2), so we can neglect the latter. Equation (2.3) simply defines the scalar field and can be solved setting $V(\phi) \partial_{v} \chi=\partial_{v} \varphi$ and $V(\phi) \partial_{u} \chi=-\partial_{u} \varphi$. Using $\varphi$ the remaining equations read

$$
\begin{aligned}
& \partial_{u} \partial_{v} \phi+\rho \bar{V}(\phi)=0, \\
& \rho \partial_{u}\left(\frac{\partial_{u} \phi}{\rho}\right)=\gamma V^{-1}(\phi)\left(\partial_{u} \varphi\right)^{2}, \\
& \rho \partial_{v}\left(\frac{\partial_{v} \phi}{\rho}\right)=\gamma V^{-1}(\phi)\left(\partial_{v} \varphi\right)^{2},
\end{aligned}
$$

where we allow for the presence of a dilatonic potential $\bar{V}(\phi)$. Equation (3.1) does not depend on $\varphi$. Hence, only the constraints (3.2, 3.3) are modified by the presence of the scalar field. The validity of the Birkhoff theorem is thus related to the r.h.s. of the Eqs. (3.2,3.3). Further, a static solution is obtained only when the r.h.s. of Eq. (3.2) is equal to the r.h.s. of Eq. (3.3). In that case $\varphi \equiv \varphi(u+v)$ or $\varphi \equiv \varphi(u-v)$ and the equations of motion can be reduced to a system of ordinary differential equations. Clearly, the above condition is satisfied in the case of pure dilaton-gravity when $\varphi$ is identically zero but cannot be satisfied by any dilaton-gravity theory coupled to a scalar field. Finally, the integrability property of the system does not seem to be related to the existence of non-static solutions.

\section{ACKNOWLEDGMENTS}

We are indebted to Vittorio de Alfaro, Alexandre T. Filippov, Larry Ford, and Alexander Vilenkin for interesting discussions and useful suggestions on various questions connected to the subject of this paper. This work has been partially supported by the grant No. 3229/96 of the University of Torino and by a Human Capital and Mobility grant of the European Union, contract number ERBFMRX-CT96-0012. 
[1] See for instance: E. Witten, Phys. Rev. D44, 314 (1991); H. Kastrup and T. Thiemann, Nucl. Phys. B425, 665 (1994); D. Marolf, Phys. Rev. D53, 6979 (1996); K.V. Kuchař, Phys. Rev. D50, 3961 (1994); C. Callan, S. Giddings, J. Harvey, and A. Strominger, Phys. Rev. D45, 1005 (1992); J. Russo, L. Susskind, and L. Thorlacius, Phys. Rev. D46, 3444 (1992); S. Carlip, J. Gegenberg, and R.B. Mann, Phys. Rev. D51, 6854 (1995); A. Barvinskii and G. Kunstatter, Phys. Lett. B389, 231 (1996); M. Banados, M. Henneaux, C. Teitelboin, and J. Zanelli, Phys. Rev. D48, 1506 (1993); J.D. Barrow, A.B. Burd, and D. Lancaster, Class. Quantum Grav. 3, 551 (1996) and references therein.

[2] See for instance: H. Nicolai, D. Korotkin, and H. Samtleben, "Integrable Classical and Quantum Gravity", Lectures given at NATO Advanced Study Institute on Quantum Fields and Quantum Space Time, Cargèse, France, 22 July - 3 August 1996, e-Print Archive: hep-th/9612065; E. Benedict, R. Jackiw, and H.-J. Lee, Phys. Rev. D54, 6213 (1996); D. Cangemi, R. Jackiw, and B. Zwiebach, Ann. Physics (N.Y.) 245, 408 (1995); K.V. Kuchař, J.D. Romano, and M. Varadarajan, Phys. Rev. D55, 795 (1997); D. Louis-Martinez, J. Gegenberg, and G. Kunstatter, Phys. Lett. B321, 193 (1994) and references therein.

[3] A.T. Filippov, Mod. Phys. Lett. A11, 1691 (1996); Int. J. Mod. Phys. A12, 13 (1997).

[4] T. Kloesch and T. Strobl, Class. Quantum Grav. 13, 965 (1996); Class. Quantum Grav. 14, 2395 (1997); Class. Quantum Grav. 14, 1689 (1997).

[5] For a deep discussion of the asymptotic structure of $2+1$ cylindrical gravity coupled to a massless scalar field see A. Ashtekar, J. Bičák, and B.G. Schmidt, Phys. Rev. D55, 687 (1997).

[6] K.C.K. Chan and R.B. Mann, Phys. Rev. D50, 6385 (1994) and Erratum, Phys. Rev. D52, 2600 (1995); Phys. Lett. B371, 199 (1996).

[7] D. Zwillinger, Handbook of Differential Equations (Boston, Academic Press, 1989), pp. 59 and 370.

[8] See for instance: Bateman Manuscript Project, Tables of Integral Transforms, ed. A. Erdélyi et al. (Mc Graw-Hill Book Company Inc., 1954) Vol. I, p. 43, formula (1). 